\title{
AN EXTENSION OF A THEOREM BY \\ C. MIRANDA IN WEIGHTED SPACES
}

\author{
Anna CAnale, Loredana Caso And Maria Transirico
}

\begin{abstract}
This paper is a continuation of [4] and extends the well-known theorem of C. Miranda (Ann. Mat. Pura Appl. (4) 61 (1963)) concerning a priori estimates of weak solutions of linear elliptic equations with discontinuous coefficients in a bounded open subset of $R^{n}$. In [4] we derive such estimates in weighted spaces considered over an arbitrary open subset of $R^{n}$. In this paper we obtain the same result replacing a condition on the coefficients by a considerably weaker one.
\end{abstract}

Mathematics subject classification (2000): 35J25, 46E35.

Key words and phrases: Elliptic equations, a priori estimates, weighted spaces.

\section{REFERENCES}

[1] V. BenCI AND D. FoRTUNATO, Weighted Sobolev spaces and the nonlinear Dirichlet problem in unbounded domains, Ann. Mat. Pura Appl. 121 (4) (1979), 319-336.

[2] A. Canale, L. CASO AND P. DI GiRonimo, Weighted norm inequalities on irregular domains, Rend. Accad. Naz. Sci. XL Mem. Mat. 16 (11) (1992), 193-209.

[3] A. CANALE, L. CASO AND P. DI GIRONIMO, Variational second order elliptic equations with singular coefficients, Rend. Accad. Naz. Sci. XL Mem. Mat. 17 (1) (1993), 113-128.

[4] A. Canale, L. Caso AND M. TRansirico, Bounds for weak solutions of elliptic equations in weighted spaces, Ricerche Mat. (to appear).

[5] A. CANALE, M. LONGOBARDI AND G. MANZO, Existence and uniqueness results for second order elliptic equations in unbounded domains, Rend. Accad. Naz. Sci. XL, Mem. Mat. 18 (1) (1994), 171-187.

[6] L. Caso And M. TRansirico, Some remarks on a class of weight functions, Comment. Math. Univ. Carolin. 37, 3 (1996), 469-477.

[7] L. CASO AND M. TRANSIRICO, The Dirichlet problem for second order elliptic equations with singular data, Acta Math. Hungar. 76 (1-2) (1997), 1-16.

[8] D. Fortunato, Spazi di Sobolev con peso ed applicazioni ai problemi ellittici, Rend. Accad. Sc. Fis. Mat. di Napoli 41 (4) (1974), 245-289.

[9] S. Matarasso and M. Troisi, Teoremi di compattezza in domini non limitati, Boll. Un. Mat. Ital. 18-B (5) (1981), 517-537.

[10] C. MIRANDA, Alcune osservazioni sulla maggiorazione in $L^{v}$ delle soluzioni deboli delle equazioni ellittiche del secondo ordine, Ann. Mat. Pura Appl. 61 (4) (1963), 151-169.

[11] R. SchianchI, Spazi di Sobolev dissimmetrici e con peso, Rend. Accad. Sc. Fis. Mat. di Napoli 42 (4) (1975), 349-388.

[12] E. M. SteIn, Singular integrals and differentiability properties of functions, Princeton Univ. Press, Princeton, New Jersey, 1970.

[13] M. TroisI, Teoremi di inclusione negli spazi di Sobolev con peso, Ricerche Mat. 18 (1969), 49-74.

[14] M. TroIsI, Su una classe di funzioni peso, Rend. Accad. Naz. Sci. XL Mem. Mat. 10 (11) (1986), $141-152$

[15] M. TroisI, Su una classe di spazi di Sobolev con peso, Rend. Accad. Naz. Sci. XL, Mem. Mat. 10 (15) (1986), 177-189.

[16] W. P. ZIEMER, Weakly differentiable functions, Springer - Verlag (1989). 